\title{
Response of the Separated Flow over an Airfoil to a Short- Time Actuator Burst
}

\author{
Xuanhong $\mathrm{An}^{1}$ and David R. Williams ${ }^{2}$ \\ Illinois Institute of Technology, Chicago IL 60616, USA \\ Andre Fernando de Castro da Silva ${ }^{3}$ and Tim Colonius ${ }^{4}$ \\ California Institute of Technology, Pasadena CA 91125 \\ and \\ Jeff D. Eldredge ${ }^{5}$ \\ University of California Los Angeles, Los Angeles CA 90095, USA
}

\begin{abstract}
Experimental measurements of the flow structure evolving in the separated flow over an NACA 0009 wing at $12^{\circ}$ angle of attack were obtained with particle image velocimetry, surface pressures, and force transducer measurements of the lift coefficient and pitching moment coefficient. Phase-averaged two-dimensional velocity field measurements provide details of the separated shear layer evolution following a four-pulse burst sequence from a synthetic jet actuator. The flow field development is quite similar to the observations made by Brzozowski, et al. (2010), who used a pulsed-combustion actuator that is orders of magnitude stronger than the synthetic jet. Proper orthogonal decomposition of the PIV data sets showed that the combination of the time-varying coefficients modes 1 and 2 correlate with the negative of the lift coefficient response. The surface pressure signals were correlated with the roll up and convection of the large-scale vortex structure that follows the actuator burst input. A spatially localized region of high pressure occurs below and slightly behind a "kink" that forms in the shear layer. A localized region of high surface pressure that follows the kinked region correlates with the lift reversal that occurs within $2.0 t^{+}$after the burst signal was triggered.
\end{abstract}

\section{Introduction}

$\mathrm{U}_{\mathrm{d}}^{\mathrm{N}}$ NDERSTANDING the dynamic behavior of separated flows in response to actuator input is essential for the development of effective active flow control systems. Ideally, we would like to be able to control the forces and moments acting on aircraft wings at time scales shorter than one convective time, where the convective time is the mean aerodynamic chord divided by the flow speed. In gust alleviation experiments, Kerstens, et al. [1] showed that the control response to actuator input was approximately four convective times. A time delay between the input disturbance and the lift response results from the interaction of multiple instabilities within the separated flow region. Using continuous harmonic actuation, Raju, et al. (2008)[2] identified three important time scales in the separated flow, which are associated with the shear layer, the separation bubble, and the wake. A different approach to studying the dynamics of the separated region is to use impulse-like disturbances to perturb the separated flow region, and follow the development of the disturbance. The impulse-like disturbances excite a broad spectrum of the separated flow, which triggers multiple instabilities. Amitay \& Glezer [3] used this approach, and identified an initial lift reversal following a short burst input from a synthetic jet actuator. The lift reversal is a key feature of the transient response

\footnotetext{
${ }^{1}$ Research Assistant, MMAE Dept., Chicago IL 60616; xan2@hawk.iit.edu, and AIAA Student Member.

${ }^{2}$ Professor, MMAE Dept., Chicago IL 60616; david.williams@iit.edu, and Associate Fellow AIAA.

${ }^{3}$ Research Assistant, Mech. and Civil Engr. Dept., Pasadena CA 91125; addasilv@caltech.edu, and AIAA Student Member.

${ }^{4}$ Professor, Mech. and Civil Engr. Dept., Pasadena CA 91125; colonius@ caltech.edu, and Associate Fellow AIAA.

${ }^{5}$ Professor, MAE Dept., Los Angeles CA 90095; eldredge@seas.ucla.edu, and Associate Fellow AIAA.
} 
at the onset of actuation. The initial decrease in lift is a characteristic of non-minimum phase behavior of a system. From a control theory perspective this means the system will have an inherent time delay that will limit the bandwidth of control, and there will be an upper limit to how fast the lift can be controlled. In fact, it was shown by Kerstens, et al. [1], that this time delay limited the bandwidth in the gust alleviation experiments.

It is now recognized that the fluid dynamic time delays inherent in separated flows are the limiting factor in the speed at which pneumatic active flow control systems can change the lift and pitching moments. One caveat is that mechanical control surfaces, such as, elevators and flaps can modify the lift at faster time scales by changing the geometry of the airfoil. However, for pneumatic actuators like synthetic and pulsed-blowing jets a different approach must be found to achieve sub-convective time scale control. Faster actuators will not make the flow respond faster. To increase the speed at which the forces can be controlled, a deeper understanding of the fluid dynamics responsible for the non-minimum phase (lift reversal) behavior of the separated flow system is required.

Detailed measurements of the separated flow evolution over a wing following a short-burst disturbance were conducted to obtain better insight into the mechanisms responsible for the lift reversal and lift enhancement. Velocity field measurements with PIV are analyzed using POD methods and Lagrangian vortex identification techniques. Four chordwise aligned surface pressure sensors were used to correlate the surface pressure distribution with the flow structures observed in the PIV measurements. The next section provides some details of the experimental arrangement. Results and a preliminary discussion are provided in Section III. The conclusions are given in Section IV.

\section{Experimental Setup}

The experiments were conducted in the Andrew Fejer Unsteady Flow Wind Tunnel at Illinois Institute of Technology. The wind tunnel has cross-section dimensions $600 \mathrm{~mm} \times 600 \mathrm{~mm}$. A nominally two-dimensional NACA0009 wing with a wingspan $b=596 \mathrm{~mm}$ and chord length $\mathrm{c}=245 \mathrm{~mm}$ was used as the test article (Fig. 1). Eight piezoelectric (zero net mass) actuators were installed in the leading edge of the wing. The slots of the actuators were located $0.05 \mathrm{c}$ from the leading edge with an exit angle of 30 degrees from the surface on the suction side of the wing.

The test freestream speed was $U_{\infty}=3 \mathrm{~m} / \mathrm{s}$, corresponding to a convective time $t_{\text {convect }}=\frac{c}{U_{\infty}}, t^{+}=\frac{t}{t_{\text {convect }}}$, and chord-based Reynolds number 49,000. The angle of attack of the wing was fixed at $\alpha=12^{\circ}$.

Surface pressure measurements were made with All-Sensors D2-P4V Mini transducers built into the wing. The pressure range for these sensors was $+/-1$ inch water. The locations of the four pressure sensors are shown in Fig. 1 as $\mathrm{P} 1-\mathrm{P} 4$.

Forces and moments were measured with an ATI NANO-17 force balance located inside the model at $30 \%$ of the chord, which is at the center of gravity of the wing. The flow field measurements (PIV) were performed $0.19 \mathrm{~b}$ away from the centerline (indicated by the orange line in Fig. 1). The 2-D time-resolved PIV data window is shown in Fig. 2 with green color, the small red circle denotes the streamwise location of the actuators and the black dots are the locations of the pressure sensors. The time resolution for the phased-averaged PIV measurement is $0.005 \mathrm{~s}\left(0.0625 t^{+}\right)$, which resulted in 800 phases covering $4 \mathrm{~s}\left(50 t^{+}\right)$. The phase averaging was done by averaging 100 flow field images for each phase.

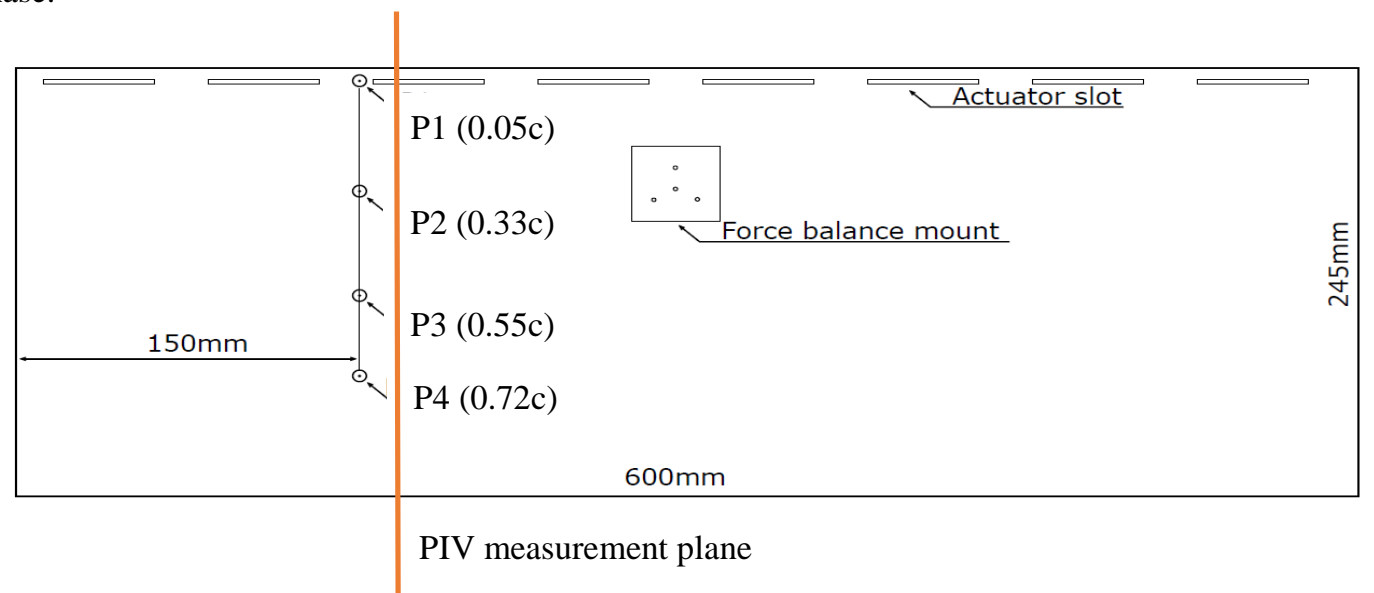

Figure 1. Top view of the wing. 


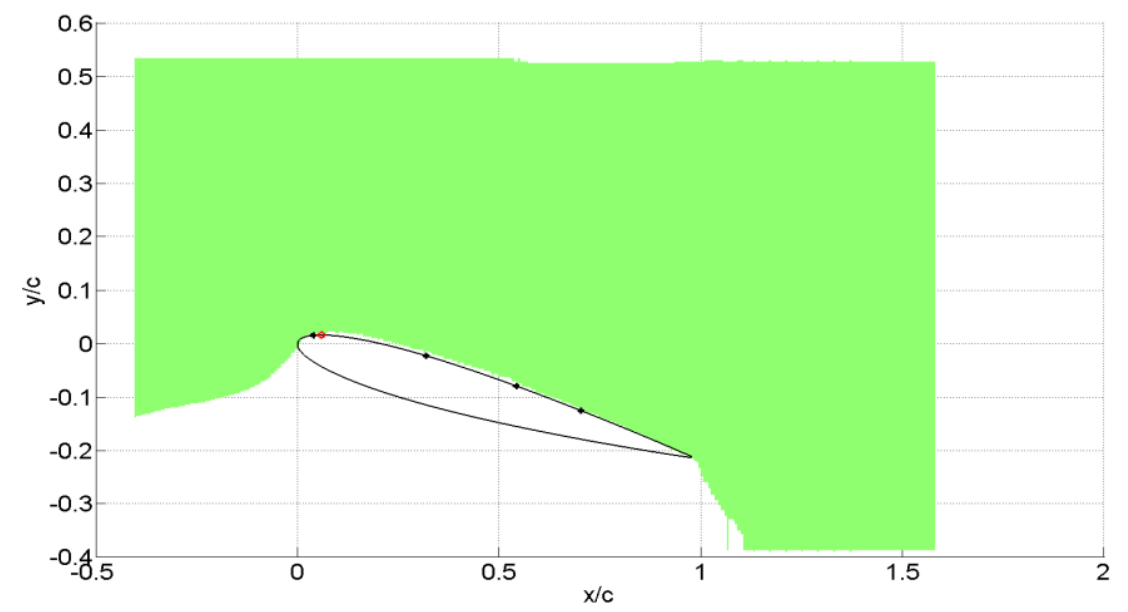

Figure 2. PIV data window.

To produce the maximum exit jet velocity, the zero net mass actuators are operated at the mechanical resonance frequency of the actuator, $f_{r}=400 \mathrm{~Hz}$ and 60 Volts amplitude. A second burst signal with $\Delta t_{p}=0.12 t^{+}$was used to modulate the resonant signal. Therefore, the actual input signal to the actuators is a short burst signal containing 4 high frequency $(400 \mathrm{~Hz})$ pulses (Fig. 3). The corresponding peak exit jet velocity measured with a hot-wire anemometer at the actuator exit is $4.9 \mathrm{~m} / \mathrm{s}$.

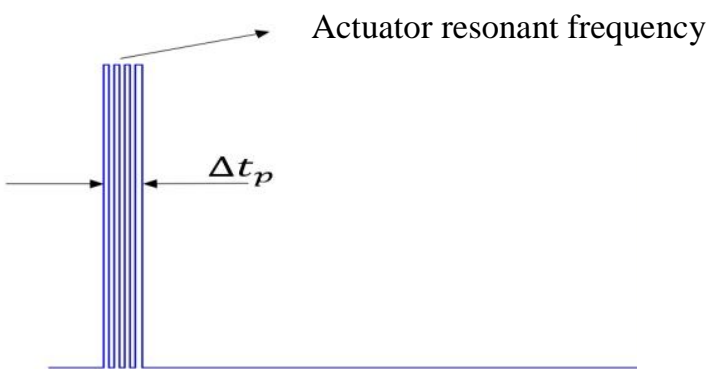

Figure 3. Input signal to the actuators is a short burst consisting of four high frequency pulses from the actuator

\section{Result and Discussion}

\section{A. Flow Field Evolution for Short Burst Actuation}

The velocity field response to a single burst is shown at different instances in time in Fig.4. The color corresponds to velocity magnitude. Insets at the lower left side of each figure show the corresponding lift coefficient response. A vertical red line shows the instant on the lift coefficient (blue) that corresponds to the velocity field. The baseline flow on the suction side is fully separated at $12^{\circ}$ angle of attack (Fig. 4a). The single burst actuation was triggered at $0 t^{+}$ and the pulse width is $0.12 t^{+}$. Reattachment of the separated flow starts to be seen at $0.5 t^{+}$from the leading edge (Fig. 4b). The reattachment produces a "kink" in the shear layer that bounds the separated flow region. The reattached region grows from the leading edge towards the trailing edge. This flow field evolution process can be seen from Fig. $4 \mathrm{~b}$ to Fig. 4e. From the inset time series lift coefficient plot in Fig. 4.2, one observes that $1.4 t^{+}$is a minimum in the lift coefficient. The lift coefficient decreases before $1.4 t^{+}$, and then increases after $1.4 t^{+}$. The lift reversal phenomenon 
was identified first by Amitay \& Glezer [3]. Since then the effect has been observed by numerous other investigators, e.g., refs $[4,5,6]$, and is now an established feature of the separated flow dynamics. The lift coefficient reaches its maximum between $2.8 t^{+}$and $4.0 t^{+}$(Fig. $4 \mathrm{~d}$ and Fig. $4 \mathrm{e}$ ), which corresponds to the reattachment point approaching the trailing edge of the wing. The flow relaxes to its baseline separated state after $10 t^{+}$. The pitching moment variation will be discussed in more detail in the later section using pressure measurement and vortex evolution.
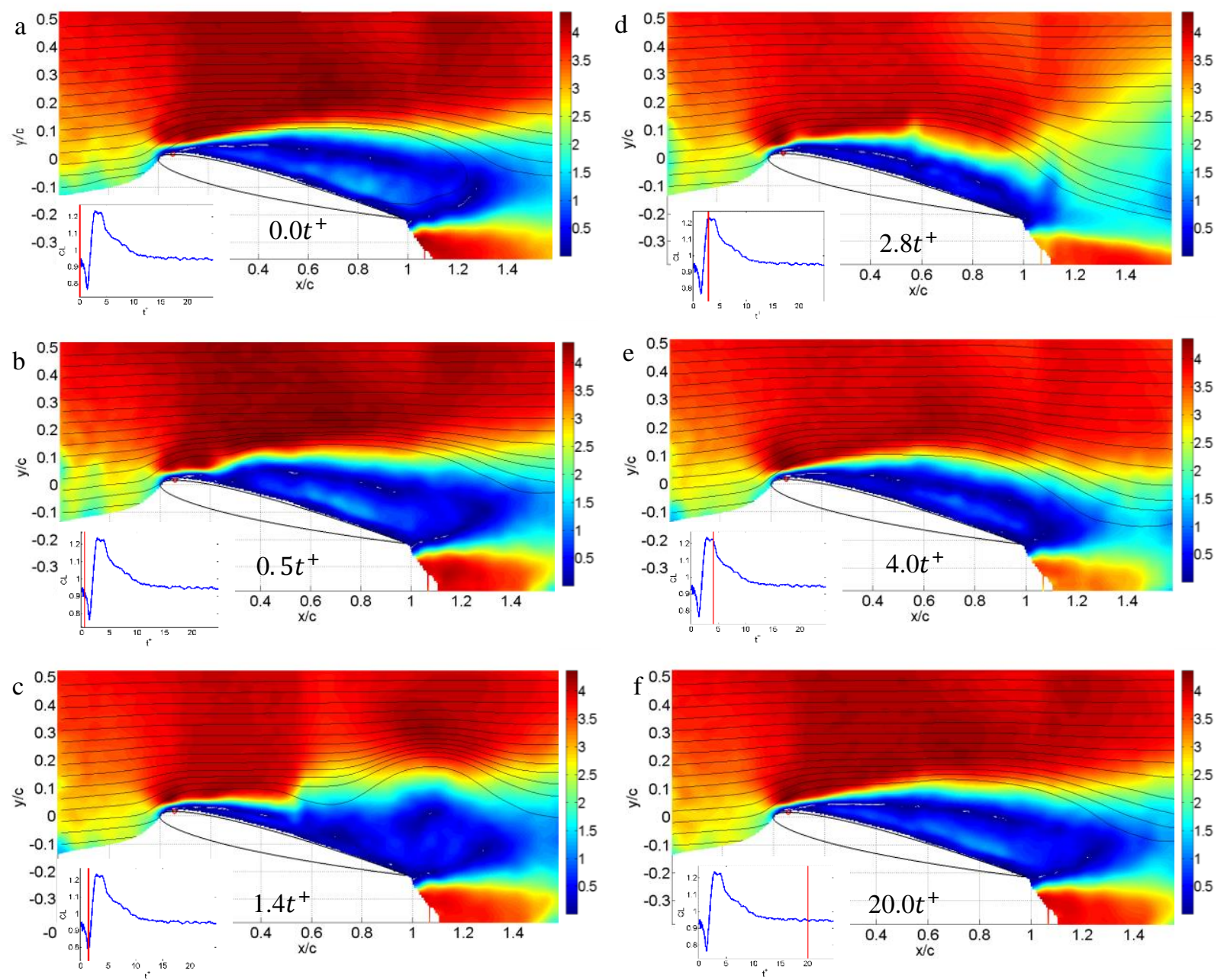

Figure 4. Phase-averaged velocity magnitude $\left(\sqrt{U^{2}+V^{2}}\right)$ and streamline time sequence plots for a single pulse actuation triggered at $0 \mathrm{t}^{+}$. The red circle on the leading edge denotes the streamwise location of the actuators. The small figure at bottom left on each flow field plot shows time series CL data (blue) and the instant (red) where the flow field measurement was taken.

Proper Orthogonal Decomposition (POD) [7] was performed on the velocity field data. This method is to reduce a large number of interdependent variables to a much smaller number of uncorrelated modes, while retaining as much as possible of the variation in the original variables [8].

$$
v(x, t)=\sum_{i=1}^{\infty} a_{i}(t) \varphi_{i}(x)
$$


In eqn. $1 a_{i}$ is the time dependent coefficient and $\varphi_{i}(x)$ is the POD basis function.

Singular Value Decomposition (SVD) [8] is performed on both horizontal velocity (U) and vertical velocity (V) from the PIV measurement. For any given $(m \times n)$ matrix $\mathrm{X}$

$$
X=U S V^{T}
$$

where $\mathrm{U}$ is an $(m \times m)$ orthonormal matrix containing the left singular vectors, $\mathrm{S}$ is a $(m \times n)$ pseudo- diagonal and semi-positive definite matrix with diagonal elements $\delta_{i}$, and $\mathrm{V}$ is an $(n \times n)$ orthonormal matrix containing the right singular vectors.

The normalized energy plot in Fig. 5 shows that most of the flow energy is contained in the first 4 POD modes, which means the first four POD modes can reconstruct a flow field containing most of the energetic structures in the actual flow field. The corresponding basis functions and the time dependent coefficients for the first four modes are plotted in Fig. 6.

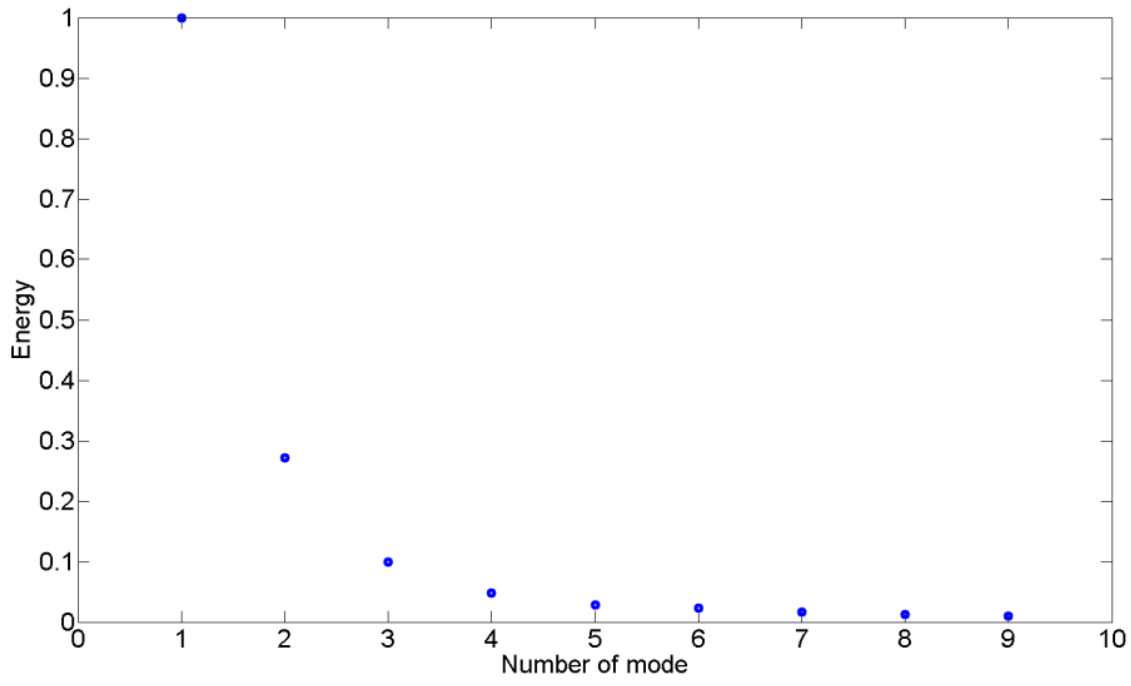

Figure 5. Energy distribution versus POD mode number. 
Mode 1
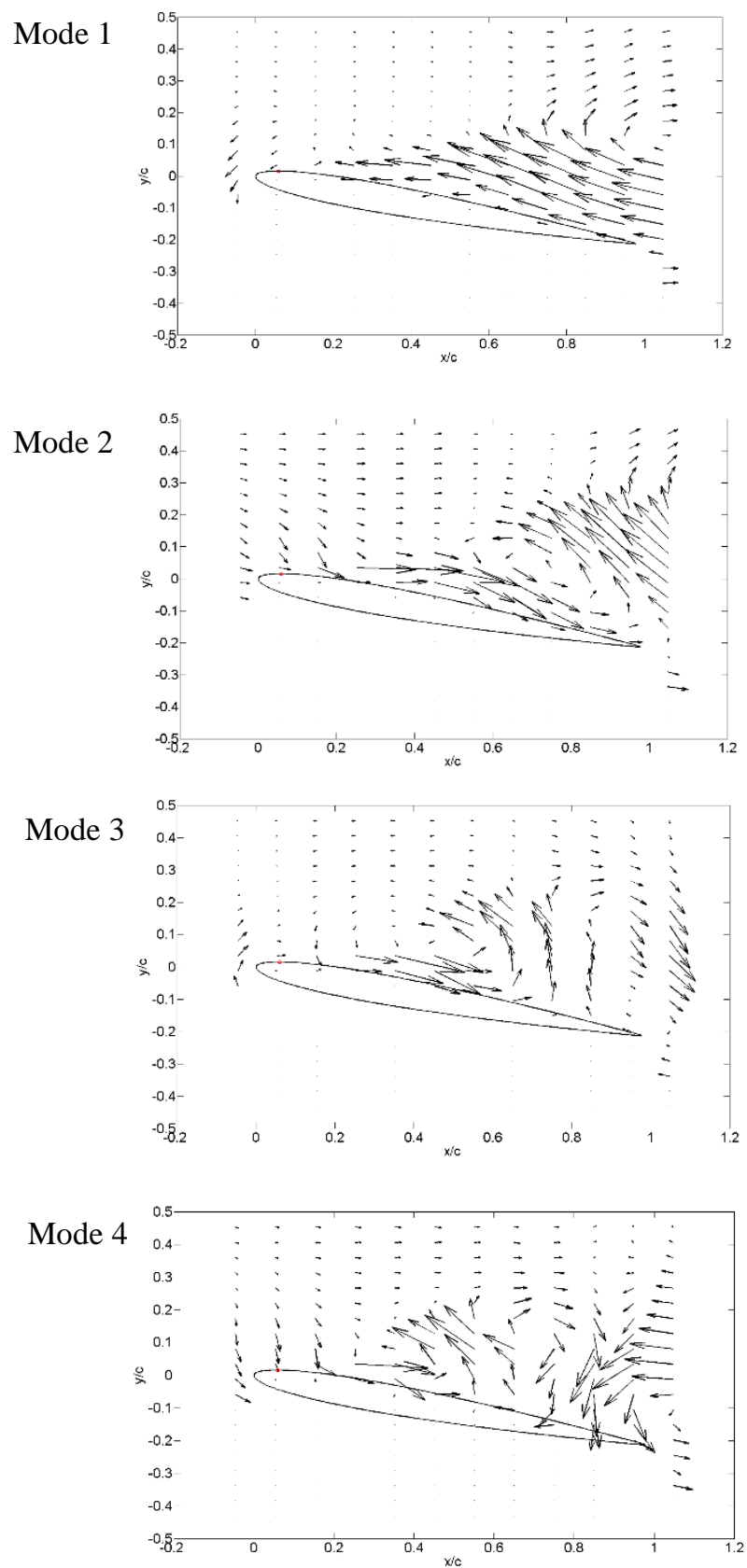
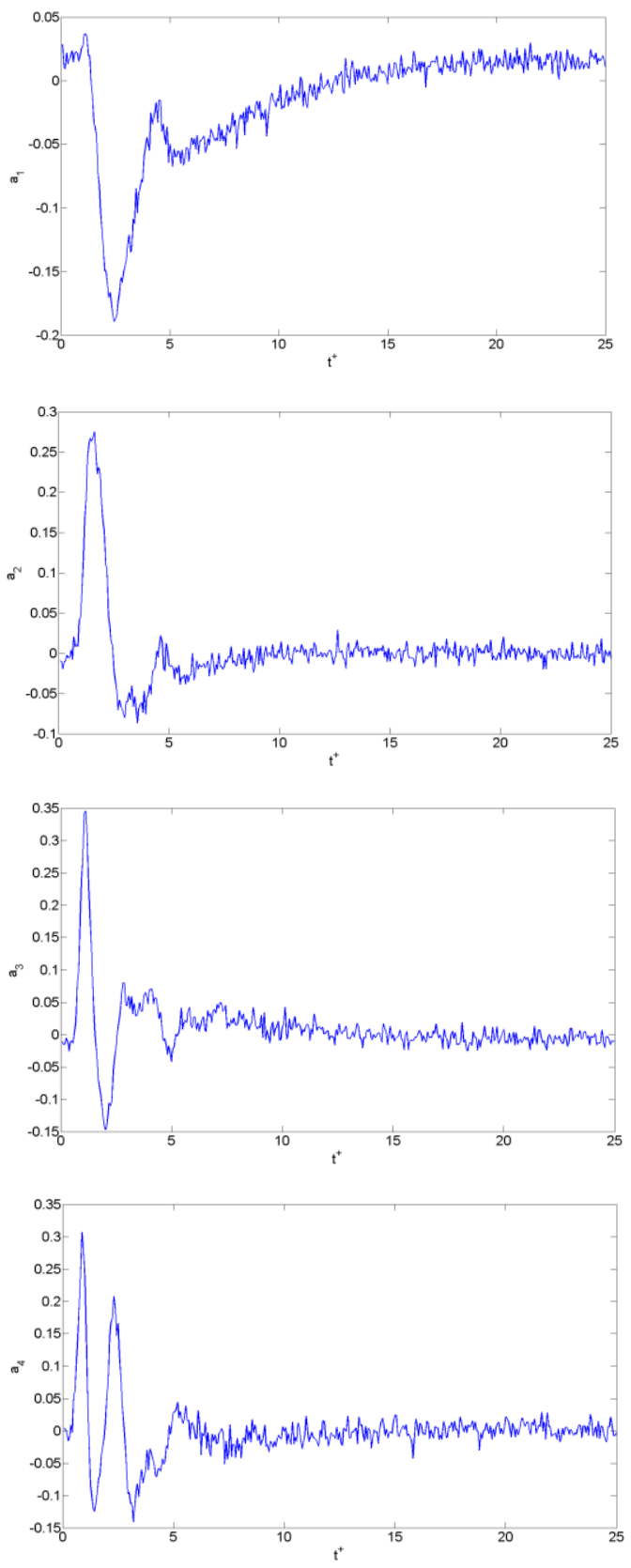

Figure 6. Comparison of the first four spatial POD modes and their corresponding temporal coefficients

In a similar experiment, Monnier, et al. [9] observed that the temporal coefficient of the second POD mode correlated with the negative of the lift coefficient variation following a single pulse input. In the current experiment we found that the flow field reconstructed by superposing the first and second modes provides a more accurate description of the vortex evolution. The sum of the temporal coefficients of mode 1 and mode 2 is plotted (Fig. 7). 
Comparing the $\triangle C L$ (baseline CL subtracted from the transient CL) to $a_{1}+a_{2}$, the combined time dependent coefficient tracks the negative $\Delta C L$ quite well, the correlation coefficient between $\Delta C L$ and $-\left(a_{1}+a_{2}\right)$ is 0.8774 .

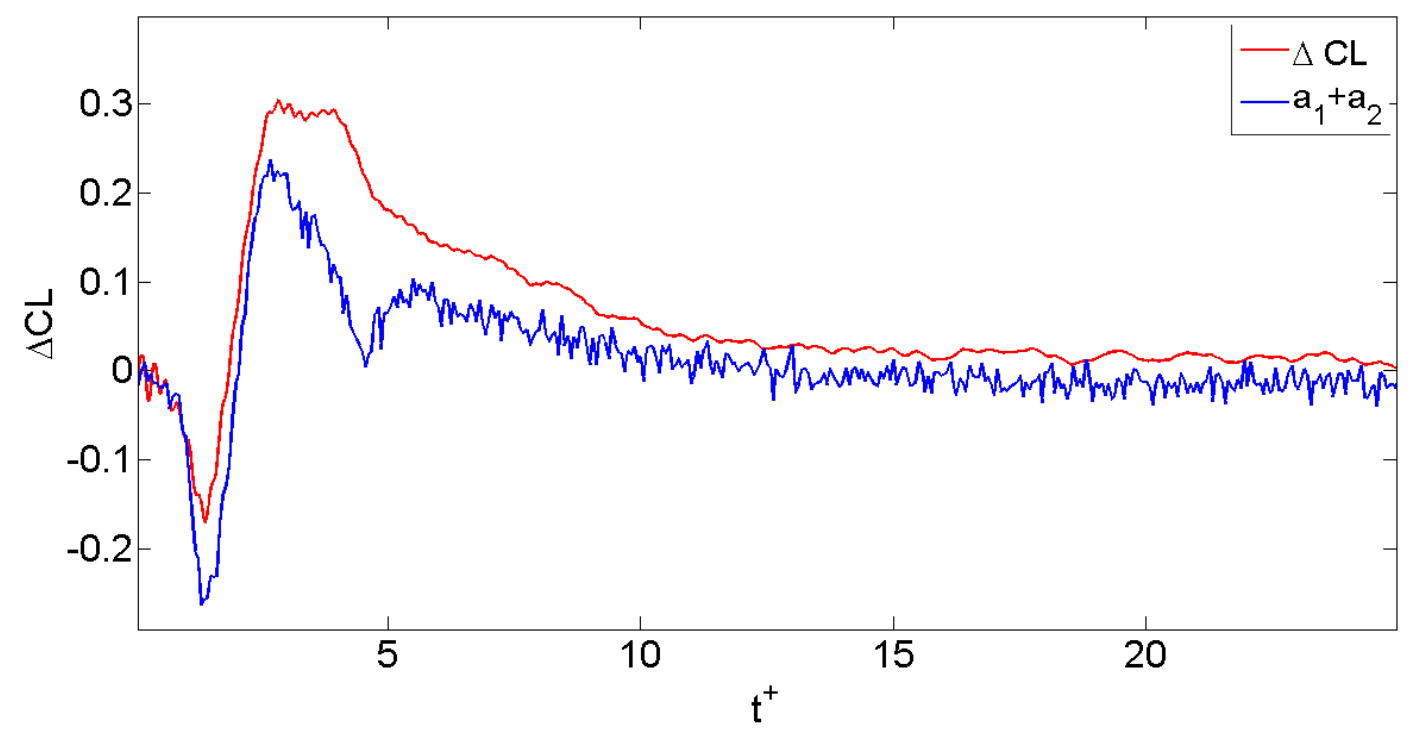

Figure 7. Time sequence of $a_{1}+a_{2}$ and $\Delta C L$ measured by the force transducer.

\section{B. CL, CM, Pressure and Vortex Structure}

The time series variation of the lift coefficient, pitching moment coefficient and the four pressure measurements are shown in Fig. 8. By contrast to the lift and pitching moment data, which are integral quantities, the surface pressure data provides additional insight into the spatial and temporal distribution of the disturbed shear layer. Therefore, pressure response to the short burst actuation was investigated at four chordwise locations. It is convenient to express the surface pressure by $P_{\infty}-P$, which means the pressure is the difference between the ambient pressure and the absolute pressure measured by the pressure sensor, which makes the increment of the surface pressure contribution to the positive lift increment.

The lift variation to the short burst actuation was studied by some researchers, refs. $[5,6,10]$. As previously discussed, the lift coefficient decreases before $1.4 t^{+}$from its baseline, and the magnitude of the local lift coefficient minimum is about $10 \%$ of the baseline lift value. A lift coefficient increase follows the lift reversal and reaches its peak at $2.8 t^{+}$, the maximum increment is about $30 \%$ of the baseline value (Fig. 8a). The pressure plot (Fig. 8c) gives more detail about the temporal variations of pressure measured by each sensor. The pressure measured at each location follows the same trend with a constant phase delay between each signal. This indicates that the reattached flow region is first established at the leading edge, and then convects towards the trailing edge rather than established globally on the entire airfoil at an instant. This correlates with the observations from the velocity flow field measurements shown in Fig. 4.

The minimum of the pitching moment (Fig. 8b) occurs $0.1 t^{+}$after the maximum lift coefficient reversal, but there is no evidence of a positive peak in the pitching moment when compared with the lift coefficient curve. On the other hand, the trend of the pitching moment curve follows the pressure variation at pressure sensor 4 . 
a

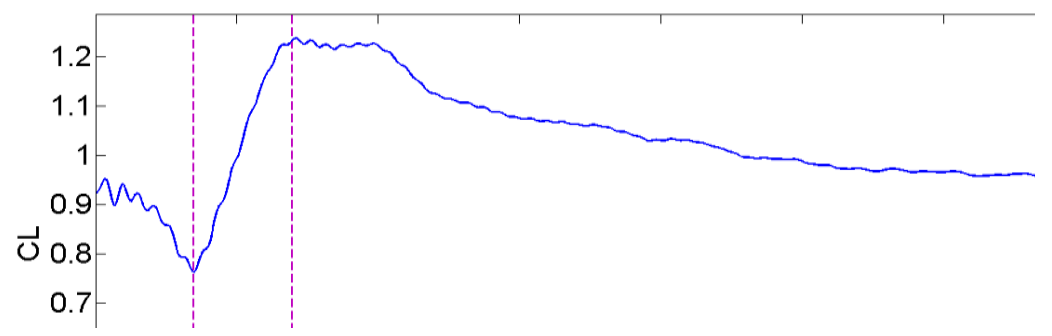

b

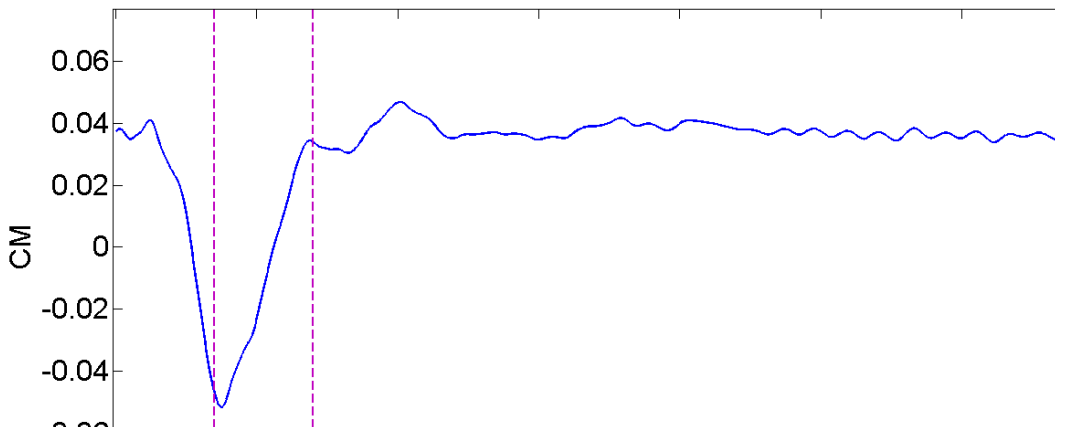

c

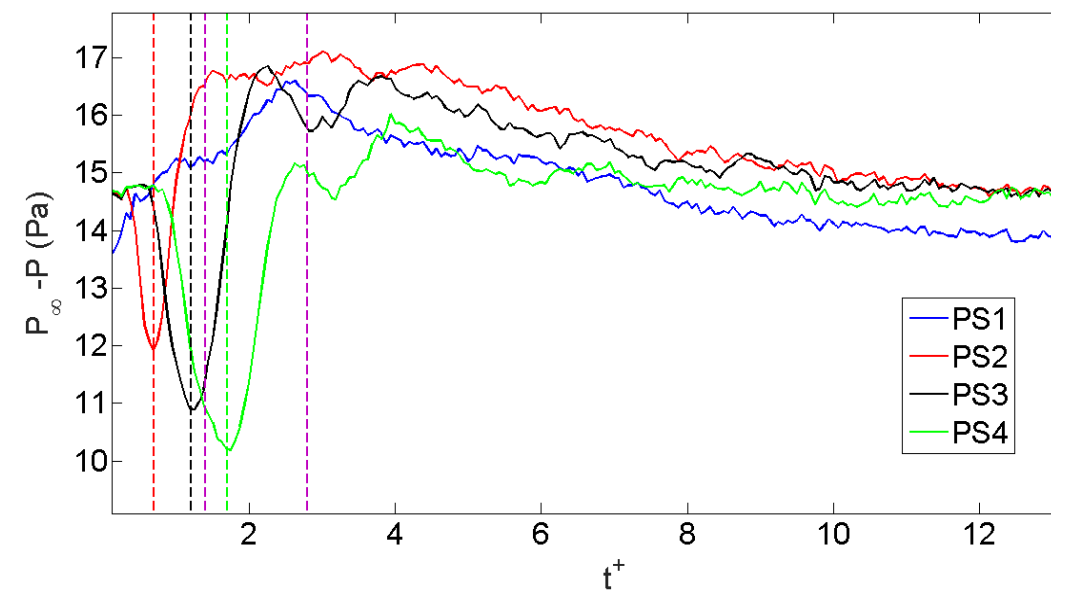

Figure 8. Lift coefficient and pitching moment measured by the force transducer, and pressures measured by the four pressure sensors. The dash lines indicate some critical instants; the minimum pressure on PS2(0. 7t $\left.t^{+}\right)$, minimum pressure on $\operatorname{PS3}\left(1.2 t^{+}\right)$, minimum $\mathrm{CL}\left(1.4 t^{+}\right)$, minimum pressure on $\operatorname{PS4}\left(1.7 t^{+}\right)$and maximum CL $\left(2.8 t^{+}\right)$, where PS stands for pressure sensor. 
Following Graftieaux, et al. [11], a Galilean invariant vortex strength $\Gamma$ in the flow was calculated using the local swirling velocity and the spatial vector relative to the center point of the calculation region. To eliminate the noise in the measured flow field, a local averaging method was used.

$$
\Gamma(P)=\frac{1}{N} \sum_{S}\left[\boldsymbol{P} \boldsymbol{M}^{\wedge}\left(\boldsymbol{U}_{M}-\widetilde{\boldsymbol{U}_{P}}\right)\right] \cdot \boldsymbol{Z}
$$

Here $\boldsymbol{P} \boldsymbol{M}$ is the spatial vector from the center point $\mathrm{P}$ to each individual point $\mathrm{M}$ surrounding $\mathrm{P}$ in the computational area $\mathrm{S}, \mathrm{N}$ is the number of points in the surrounding area $\mathrm{S}, \boldsymbol{U}_{M}$ is the velocity at the point $\mathrm{M}, \widetilde{\boldsymbol{U}}_{P}$ is the mean velocity in the area $S$ and $\boldsymbol{Z}$ is the unit vector normal to the measurement plane. But in the $2 \mathrm{D}$ case, Eq. (3) becomes

$$
\Gamma(P)=\frac{1}{N} \sum_{S}\left[\boldsymbol{P} \boldsymbol{M}^{\wedge}\left(\boldsymbol{U}_{M}-\widetilde{\boldsymbol{U}_{P}}\right)\right]
$$

To better visualize the vortex structure, the Galilean invariant $\lambda_{2}[12]$ was used to identify the vortex boundary. Only the vortex strength $\Gamma$ is shown inside the vortex boundary defined by $\lambda_{2}$.

Taking the gradient of Navier-Stokes equations, the symmetric part without unsteady and viscous effects is

$$
\Omega^{2}+S^{2}=-\frac{1}{\rho} \nabla(\nabla P)
$$

where $S=\frac{J+J^{T}}{2}, \Omega=\frac{J-J^{T}}{2}$ and $J$ is the velocity gradient tensor. Therefore, we consider only $\Omega^{2}+S^{2}$ to determine the existence of a local pressure minimum due to vertical motion. The vortex core can be defined as a connected region with two negative eigenvalues $\left(\lambda_{2}<0\right)$. In our case, $\left(\lambda_{2}<-100\right)$ is used to eliminate the turbulence effect in the freestream.

The vortex structure (Fig. 9) is plotted at each critical instant on the lift, moment and pressure curves, corresponding to the dashed lines shown in Fig. 8. The vortex structure at $0 t^{+}$(Fig. 9a) shows some small clockwise rotating vortices above the airfoil in the separated shear layer, and some counter-clockwise rotating vortices near the trailing edge indicate the trailing edge vortex. Fig. $9 \mathrm{~b}$ shows the vortex structure taken at $0.7 t^{+}$, where the pressure at pressure sensor 2 reaches its minimum. On the other hand, the leading edge vortex in the baseline separates and sheds due to the single pulse actuation. This shed vortex is then growing and shedding into the wake, while a new leading edge vortex is being established (Fig. 9c to Fig. 9e). At $2.8 t^{+}$(Fig. 9f), both the leading edge and the trailing edge vortices have shed into the wake, and resulting the flow leaves the trailing edge smoothly. This corresponds to the reattachment point reaching the trailing edge, and the lift increment reaching its maximum. Lastly, as expected, at $20 t^{+}$after the actuation (Fig. 9g) the flow has returned back to the original baseline situation.

Comparing Fig. 9 to Fig. 8, it can be seen that the negative pressure peak is moving with the middle point between the (clockwise rotating) shed vortex and the newly established leading edge vortex. The negative induced velocity by the (clockwise rotating) shed vortex cancels the newly established positive surface velocity by the reattachment process at middle point between the two vortices. Therefore, the suction side velocity becomes very small at that point (Fig. 9b, Fig. 9c and Fig. 9f), causing a local pressure drop. As a consequence, the sequence of the pressure reversals produces the lift reversal (Fig. 9d and Fig. 8).

On the other hand, there is no pressure reversal observed by pressure sensor 1 (Fig. 8c), because the leading edge vortex separation point is located behind this pressure sensor. 
a

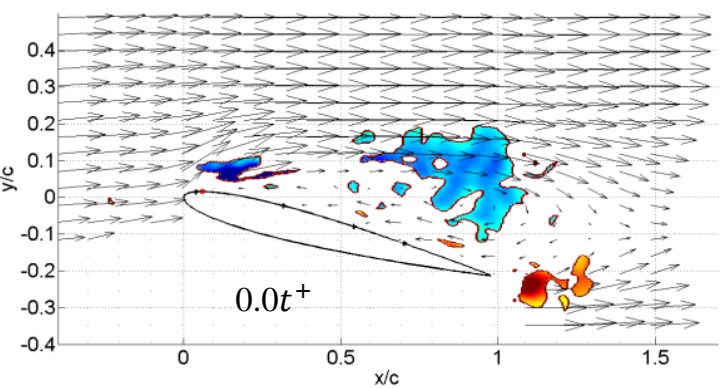

b

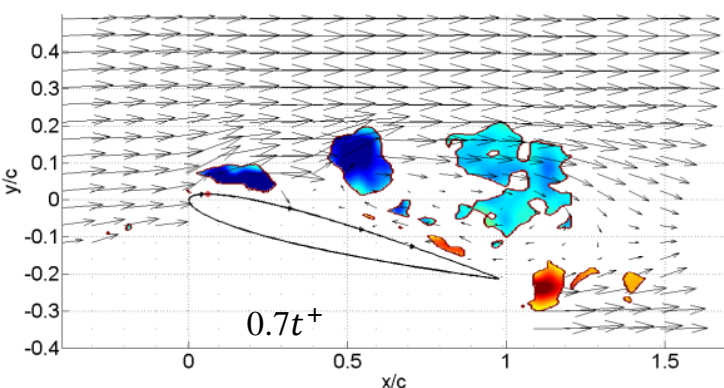

c

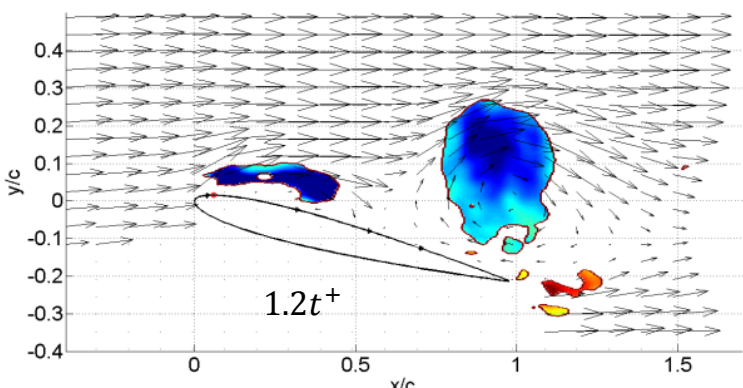

d

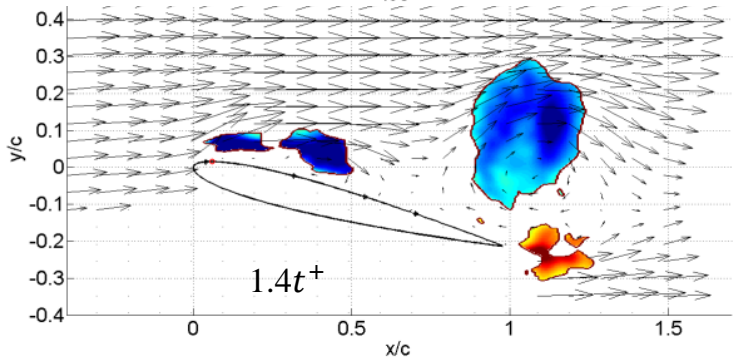

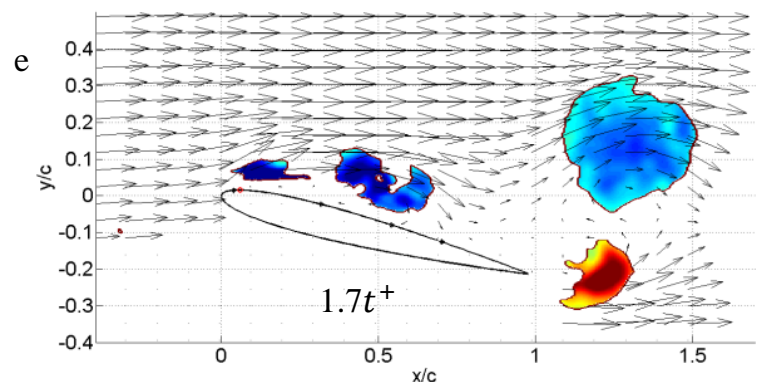
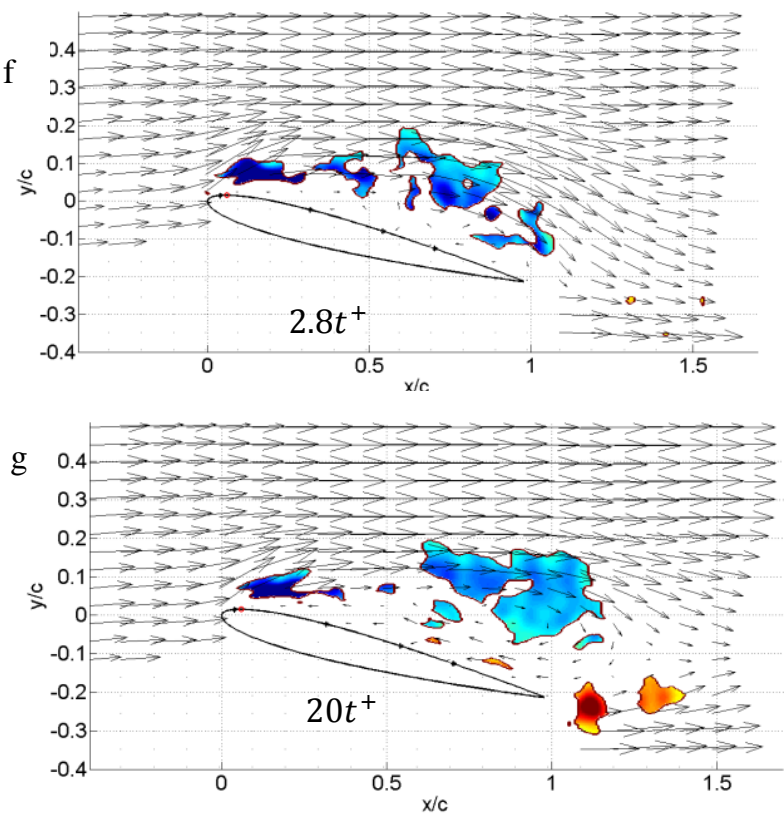

Figure 9. Vortex and velocity plot, the plots from a to $\mathrm{g}$ represent baseline $\left(0 t^{+}\right)$, minimum pressure on PS2 $\left(0.7 t^{+}\right)$, minimum pressure on PS3 $\left(1.2 t^{+}\right)$, minimum CL $\left(1.4 t^{+}\right)$, minimum pressure on PS4 $\left(1.7 t^{+}\right)$, maximum CL $\left(2.8 t^{+}\right)$ and baseline $\left(20 t^{+}\right)$. 


\section{CL, CM Estimation Using Limited Number of Pressure Measurement Points}

The previous section focuses on the relation between the flow structure and the surface pressure, which leads to a better understanding on the flow structure evolution that follows the single burst actuation disturbance. But both the force and moment response are important to flight dynamics.

To estimate the force and moment with the measured pressure data, a set of weight coefficients along with an offset are added to equation Eq.6. This method was proposed by Reissner [13]

$$
\begin{array}{r}
F=\int P d s \\
o r, \\
F=\sum P_{i} S_{i}
\end{array}
$$

where $F$ is the force and $P_{i}$ is the pressure acting on the surface with an area $S_{i}$. So equation (7) becomes

$$
F_{j}=\sum\left[P_{i j} S_{i}\right]\left[w_{i}\right]+w_{0} \cdot 1
$$

where $w$ is a weight factor (column vector) and $w_{0}$ is a constant offset. The number of elements in $w$ equals to the number of pressure sensors. Using an expanded form of $F$ and $w$, and rewriting Eq. 5 in matrix form,

$$
F_{e}=[P \cdot S] w_{e}
$$

where $F_{e}=\left[F_{j}\right], w_{e}=\left[\begin{array}{c}W \\ w_{0}\end{array}\right]$ and $P=\left[P_{i j} 1_{j}\right]$.

To be more specific, we substitute $F_{e}$ with lift $L$, which is measured by a force transducer. Then, since $P$ and $L$ can be non-square matrices

$$
w_{e}=\left[(P \cdot S)^{T}(P \cdot S)\right]^{-1}(P \cdot S)^{T} L^{T}
$$

Therefore, $w_{e}$ can be solved with some experimental data sets including both pressure and lift force. This means the lift force can be estimated using the measured pressure by Eq. 8 after $w_{e}$ is identified.

In the current research, there are only four pressure sensors on the surface of the airfoil's suction side (Fig. 1), and 4076 experimental time series data points are used to solve $w_{e}$.

This approach can also be applied on the moment estimation. The only difference is that the moment arm $r_{i}$ needs to be included. So Eq. 8 becomes

$$
F_{j}=\sum\left[P_{i j}\left(S_{i} \cdot r_{i}\right)\right]\left[w_{i}\right]+w_{0} \cdot 1
$$



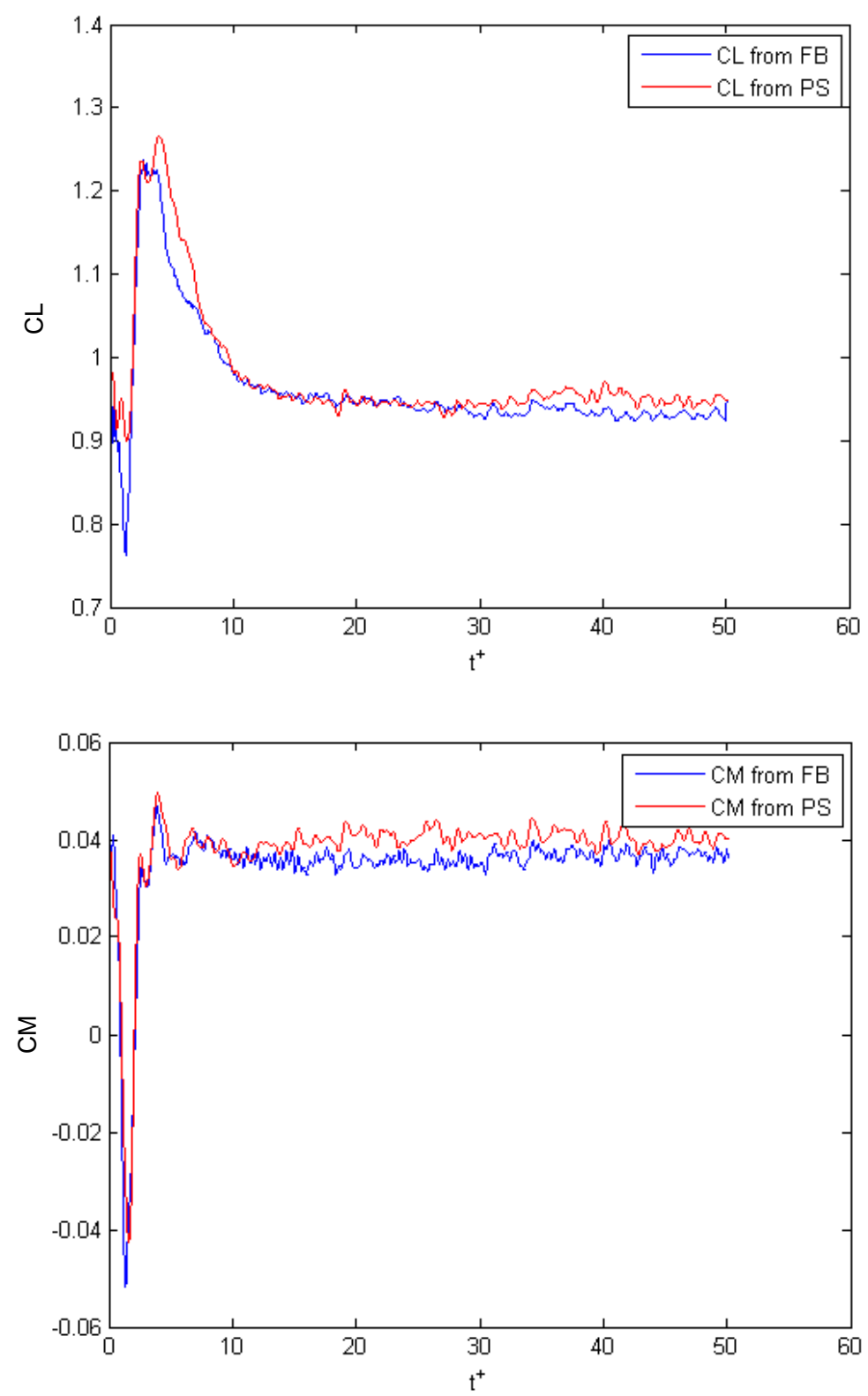

Figure 10. Comparison of lift coefficient and pitching moment coefficient directly measure by the force transducer with the values estimated by the pressure measurement.

Fig.10 shows the lift coefficient and the pitching moment coefficient estimated by the pressure measurements. The $\mathrm{CM}$ estimation using pressure fits the data measured by the force transducer very well, the CL estimation from the pressure data is reasonably good, but it fails to capture the lift reversal in the CL plot measured by the force transducer. This may suggest that more detailed structures need to be identified when the actuation is applied.

\section{Conclusions}

The evolution of the flow field's velocity and the vortex structure, pressure distributions, lift and pitching moment coefficients were documented following a short burst actuation applied to a separated flow over a wing. The 
streamlines of the flow field demonstrated the reattachment process corresponding to the lift coefficient variation. The maximum lift increment occurs at $2.8 t^{+}$when the flow was reattached. The POD analysis shows most of the energy is stored in the first 4 modes, and the combined time coefficient of POD mode 1 and mode 2 tracks the negative lift coefficient curve. Unlike the lift coefficient curve, there is no obvious positive increment observed in the pitching moment curve. There is a point of minimum velocity on the suction side of the wing moving with the clockwise rotating large-scale shed leading edge vortex, this causing a pressure drop at this moving point and contributes to a lift reversal before this vortex shedding into the wake. Despite the lift reversal under-estimation the 'weight factor' method did a reasonably good job on estimating the lift coefficient and pitching moment behavior using only 4 pressure sensors on the suction side surface of the wing.

\section{Acknowledgments}

Support by the U.S. Air Force Office of Scientific Research (FA9550-14-1-0328) with program manager Dr. Douglas Smith is gratefully acknowledged.

\section{References}

${ }^{1}$ Kerstens, W., Pfeiffer, J., Williams, D., King, R., \& Colonius, T. (2011). Closed-loop control of lift for longitudinal gust suppression at low Reynolds numbers. AIAA journal, 49(8), 1721-1728.

${ }^{2}$ Raju, R., Mittal, R., \& Cattafesta, L., (2008). Dynamics of Airfoil Separation Control. AIAA J. 46 (12) 3103-3115.

${ }^{3}$ Amitay, M., \& Glezer, A. (2002). Controlled transients of flow reattachment over stalled airfoils. International Journal of Heat and Fluid Flow, 23(5), 690-699.

${ }^{4}$ Woo, G. T., \& Glezer, A. (2013). Controlled transitory stall on a pitching airfoil using pulsed actuation. Experiments in Fluids, 54(6), 1-15.

${ }^{5}$ Brzozowski, D. P., K. Woo, G. T., Culp, J. R., \& Glezer, A. (2010). Transient separation control using pulse-combustion actuation. AIAA journal, 48(11), 2482-2490.

${ }^{6}$ Monnier, B., Williams, D. R., Weier, T., \& Albrecht, T. (2015). Comparison of a Separated Flow Response to Localized and Global-type Disturbances. In53rd AIAA Aerospace Sciences Meeting (p. 1056).

${ }^{7}$ Kosambi, D. D. (1943). Statistics in function space. J. Indian Math. Soc,7(1), 76-88.

${ }^{8}$ Kerschen, G., Golinval, J. C., Vakakis, A. F., \& Bergman, L. A. (2005). The method of proper orthogonal decomposition for dynamical characterization and order reduction of mechanical systems: an overview. Nonlinear dynamics, 41(1-3), 147-169.

${ }^{9}$ Monnier, B., Williams, D. R., Weier, T., \& Albrecht, T. (2015). Comparison of a Separated Flow Response to Localized and Global-type Disturbances. Experiments in Fluids,57(7), 1-16.

${ }^{10}$ Cierpka, C., Weier, T., \& Gerbeth, G. (2008). Evolution of vortex structures in an electromagnetically excited separated flow. Experiments in Fluids,45(5), 943-953.

${ }^{11}$ Graftieaux, L., Michard, M., \& Grosjean, N. (2001). Combining PIV, POD and vortex identification algorithms for the study of unsteady turbulent swirling flows. Measurement Science and Technology, 12(9), 1422.

${ }^{12}$ Jeong, J., \& Hussain, F. (1995). On the identification of a vortex. Journal of fluid mechanics, 285, 69-94.

${ }^{13}$ Reissner, F. (2015). Hysteresis modeling and comparison of controller effectiveness on a pitching airfoil. Master's Thesis, technische universität berlin. 